\title{
Evaluación del estigma hacia pacientes con cáncer: una aproximación psicométrica
}

\section{Assessment of stigma toward cancer patients: A psychometric approach}

\author{
Julio C. Penagos-Corzo y Sheila Pintado Cucarella ${ }^{1}$
}

\begin{abstract}
Citación: Penagos-Corzo, J.C. y Pintado-Cucarella, S. (2020). Evaluación del estigma hacia pacientes con cáncer: una aproximación psicométrica. Psicología y Salud, 30(2), 153-160. https:// doi.org/10.25009/pys.v30i2.2650.
\end{abstract}

\section{RESUMEN}

\begin{abstract}
El objetivo del presente estudio fue determinar las propiedades psicométricas de un cuestionario para evaluar el estigma hacia personas con cáncer, en una muestra de 1340 estudiantes universitarios mexicanos. El análisis factorial exploratorio indicó tres elementos: Discriminación, Imposibilidad de recuperación y Estereotipos para pacientes con cáncer. La consistencia interna del instrumento indica una adecuada confiabilidad para el total del instrumento. El análisis factorial confirmatorio arrojó un óptimo ajuste, lo que sugiere su idoneidad para evaluar el estigma.
\end{abstract}

Palabras clave: Psicooncología; Validez; Estructura factorial; Cáncer; Actitudes estigmatizadoras.

\begin{abstract}
The objective of the present study was to determine the psychometric properties of a questionnaire designed to evaluate stigma towards people with cancer, in a sample of 1340 Mexican undergraduate students. Exploratory factor analysis indicated three factors: Discrimination, Impossibility of recovery, and Stereotypes for cancer patients. The internal consistency of the instrument indicates adequate reliability for the entire instrument. Confirmatory factor analysis yielded an optimal fit, suggesting good feasibility for assessing stigma.
\end{abstract}

Key words: Psycho-oncology; Validity; Factor structure; Cancer; Stigmatizing attitudes.

$\mathrm{L}$ a salud tiene una dimensión colectiva y biosocial que resulta indispensable entender (Lopez et al., 2017) porque, entre otras cosas, las interacciones sociales pueden tener efectos en la salud y en la enfermedad (Martínez, 2008; Napier et al., 2014), e incluso en las formas en que el paciente es tratado o en que su enfermedad es percibida. Por ejemplo, algunas personas diagnosticadas con cáncer pueden ser sujetos de estigmatización y discriminación por el hecho de padecer la enfermedad (Fujisawa y Hagiwara, 2015; Lee y Kim, 2011).

El estigma es un concepto multidimensional, caracterizado como un atributo negativo que desacredita a la persona (Parker, 2012), y puede considerársele como una actitud (Hofmann-Broussard, Armstrong, Boschen y Somasundaram, 2017). En el caso del cáncer, dicho estigma se ha asociado con la incomodidad de las personas, la evitación y hasta la discriminación financiera, entre otros (Marlow y Wardle, 2014). Se ha reportado que a las personas con cáncer se les percibe como una fuente de amenaza, lo que aumenta

\footnotetext{
${ }^{1}$ Universidad de las Américas Puebla. Ex hacienda Sta. Catarina Mártir, San Andrés Cholula. 72810 Puebla, México, tels. (222)229-20-55 y (222)229-24-86, correos electrónicos: julioc.penagos@udlap.mx y maria.pintado@udlap.mx. Artículo recibido el 19 de septiembre de 2019 y aceptado el 3 de abril de 2020.
} 
las actitudes negativas hacia ellas (Berrenberg, Finlay, Stephan y Stephan, 2002). Tales percepciones, que aparecen en diferentes poblaciones (Machado, Biancovilli y Jurberg, 2017) y rangos de edad -como por ejemplo en estudiantes universitarios (Dany, Chidiac y Nassar, 2015; Grigsby et al., 2014) - tienen efectos negativos en la salud, e incluso en quien las manifiesta. En este sentido, la resistencia observada en estudiantes universitarias en tomar parte activa en la exploración del cáncer de mama parece estar vinculada a creencias, sensación de vergüenza y otras actitudes negativas (Latif, 2014), así como al desconocimiento y la falta de interés (Boulos y Ghali, 2014; Sambanje y Mafuvadze, 2012). De hecho, aunque haya variaciones en la estigmatización por parte de diversos grupos poblacionales, la relación negativa entre ese estigma y la ejecución de exploraciones y pruebas de cribado es común en todos ellos (Vrinten, Gallagher, Waller y Marlow, 2019).

La vinculación de la muerte con la enfermedad y la ansiedad que se deriva de esta relación contribuyen asimismo a explicar dichas actitudes hacia las personas enfermas, pues el cáncer, en diferentes culturas, habitualmente se asocia con temor y recelo, y tiene una connotación negativa asociada al sufrimiento y la muerte (Peiró et al., 2006; Rodríguez y Palacios, 2013), la que es reforzada en ciertos anuncios televisivos (Chapple, Ziebland y McPherson, 2004). Así, el distanciamiento social que las personas muestran hacia el paciente, el cual este percibe como discriminación (Feng et al., 2018), puede explicarse por el miedo a morir (Mosher y Danoff-Burg, 2007), de modo que esa conducta discriminatoria quizá tiene que ver con la habilidad para lidiar con la propia mortalidad (Mosher y Danoff-Burg 2007).

Sin embargo, hay una falta de acuerdo en cómo conceptualizar y examinar el estigma del cáncer, lo que impide establecer una relación entre los hallazgos reportados sobre este tópico $(\mathrm{Fu}-$ jisawa y Hagiwara, 2015). En primera instancia, se requieren instrumentos válidos y confiables que midan el estigma y que puedan utilizarse en diferentes poblaciones. Cho et al. (2013) diseñaron un cuestionario para evaluar las actitudes hacia los pacientes con cáncer en una muestra sin historial clínico de esta enfermedad, el cual evalúa tres dominios: Imposibilidad de recuperación, Estereoti- pos y Discriminación. Los datos del referido trabajo destacan una percepción de déficits en la vida laboral y social de los pacientes con cáncer y la improbabilidad de su cura; además, nociones estereotipadas sobre la apariencia, y las capacidades y vida sexual reducidas (Cho et al., 2013).

Las implicaciones de estigmatizar a personas que padecen cáncer son diversas, y van desde mostrar conductas falsamente positivas por parte de los pacientes -cuyo fin es evitar la estigmatización (Trusson y Pilnick, 2017) y el sufrimiento que la misma provoca (Greidanus et al., 2018)-, hasta la depresión (Cho, Choi et al., 2013) y el hostigamiento laboral (Stergiou-Kita, Pritlove y Kirsh, 2016). Tales implicaciones, así como la importancia de conceptualizar y examinar el estigma del cáncer, no se reflejan en instrumentos que atiendan ampliamente esta necesidad. Por ejemplo, al momento de emprender el presente estudio fue imposible localizar un solo instrumento en lengua española que evaluara el estigma hacia pacientes con cáncer en la base de datos Psyctests con la siguiente cadena de búsqueda: [(attitude* and negative and cancer) or (stigma and cancer)]. Se hicieron búsquedas similares en Web of Science y Scopus sin lograr encontrar instrumentos traducidos o creados en lengua hispana, diseñados específicamente para evaluar el referido estigma. Para estas búsquedas, dado el carácter genérico de las bases de datos, se anexó a la cadena de búsqueda anterior: [and ("psychometric properties" or "propiedades psicométricas" or "internal consistence" or "consistencia interna" or validity or validez or reliability or confiabilidad or "factor analysis" "análisis factorial")]. Por ello, el objetivo del presente estudio transversal descriptivo y exploratorio, con medida en un único momento temporal, fue evaluar las propiedades psicométricas del cuestionario de actitudes hacia pacientes con cáncer desarrollado por Cho et al. (2013) en estudiantes universitarios.

\section{MÉTODO}

\section{Participantes}

Mediante una invitación pública hecha dentro de un campus universitario, se seleccionó una mues- 
tra conformada por 1,340 estudiantes universitarios mexicanos con una media de edad de 19.47 (D.E. $=1.536)$. De ellos, 800 (59.7\%) fueron mujeres y $540(40.3 \%)$ varones.

En cuanto a los antecedentes familiares de cáncer, 685 (51.1\%) afirmaron tenerlos (los más comunes: cáncer de mama, próstata y pulmón), frente a 655 que no los tenía (48.9\%). Se consideró como criterio de exclusión haber recibido un diagnóstico de cáncer, pues el instrumento está orientado a medir el estigma hacia los pacientes de cáncer por parte de no pacientes. Así, en la muestra original de 1,358 participantes, 18 reportaron tener o haber tenido diagnóstico de cáncer, por lo que fueron excluidos. A todos se les garantizó el anonimato y la confidencialidad de sus datos. Aquellos que aceptaron participar dieron su consentimiento informado. El trabajo se apegó al a los principios éticos para la investigación en seres humanos de la Declaración de Helsinki (World Medical Association, 2013) y fue aprobado por el Comité de Ética, que es parte del Comité de Investigación de la institución.

\section{Instrumentos}

Se utilizó el cuestionario de Cho et al. (2013), el cual consta de 12 ítems agrupados en tres categorías: imposibilidad de recuperación, estereotipos hacia el cáncer y discriminación. Las respuestas se evalúan mediante una escala Likert que va de 1 ("fuertemente en desacuerdo") a 4 ("fuertemente de acuerdo"). La consistencia interna reportada del cuestionario original es de .79 , y de .68 para la subescala "Imposibilidad de recuperación", de .60 para la de "Estereotipos hacia el cáncer"; y de .71 para la de "Discriminación".

\section{Procedimiento}

El proceso de adaptación cultural del instrumento se realizó siguiendo las recomendaciones de Reyes y García (2008) así como de las de Reichenheim y Moraes (2007). El cuestionario se tradujo mediante el seguimiento de su equivalencia semántica, operativa y de medición. Tres expertos validaron la traducción de forma independiente. Se volvió a traducir de acuerdo a los cambios sugeridos, y finalmente el instrumento final se adaptó por consenso (Tabla 1).

Tabla 1. Versión en traducida del instrumento utilizado.

\section{Imposibilidad de recuperación}

1. Es imposible tratar el cáncer a pesar de la ciencia médica altamente desarrollada.

2. Es muy difícil volver a estar sano una vez que se diagnostica el cáncer a una persona.

3. Los pacientes con cáncer no serían socialmente activos una vez diagnosticados con cáncer.

4. La capacidad de los pacientes con cáncer para realizar tareas en el lugar de trabajo puede disminuir incluso después de un tratamiento exitoso contra el cáncer.

\section{Estereotipos de pacientes con cáncer}

5. Los pacientes con cáncer son fácilmente reconocibles por su apariencia.

6. Los pacientes con cáncer tendrían dificultades para tener intimidad sexual.

7. Los pacientes con cáncer merecen ser protegidos en la sociedad.

8. Los pacientes con cáncer no podrían hacer contribuciones a la sociedad.

\section{Discriminación contra los pacientes con cáncer}

9. Me siento incómodo cuando estoy con pacientes con cáncer.

10. Tiendo a evitar interactuar con vecinos que tienen cáncer.

11. Evitaría casarme con personas cuyos familiares tienen cáncer.

12. Evitaría trabajar con personas que tienen cáncer. 
A dicho cuestionario se le incorporaron los datos sociodemográficos de los participantes, como sexo, antecedentes familiares de cáncer y edad.

Hecho lo anterior, se aplicó el instrumento en los salones de clase de forma colectiva. Previamente a la recolección de los cuestionarios, se comentaron los objetivos del estudio y siempre se dio tiempo a los participantes para aclarar sus dudas sobre el instrumento y consentimiento informado de manera individual.

\section{Análisis estadístico}

La confiabilidad del instrumento se determinó mediante el coeficiente omega (McDonald, 2011), que se ha señalado como una mejor opción que el alfa de Cronbach debido a que proporciona aproximaciones más precisas de la confiabilidad (Revelle y Zinbarg, 2009). Para los análisis exploratorio y confirmatorio, la muestra se dividió aleatoriamente en dos submuestras con aproximadamente $50 \%$ cada una. A fin de conocer la estructura factorial, se efectuó un análisis factorial exploratorio (AFE) mediante factorización de ejes principales con rotación promax en la submuestra de 695 participantes, y para probar el ajuste al modelo se condujo, en la otra muestra de 645 participantes, un análisis factorial confirmatorio (AFC), para lo cual se utilizó una estimación de máxima verosimilitud.

\section{RESULTADOS}

\section{Datos descriptivos}

El porcentaje de participantes que estuvo de acuerdo o muy de acuerdo con que es imposible tratar el cáncer a pesar de los avances médicos, fue de $21.2 \% ; 26.3 \%$ creía que, una vez diagnosticada la enfermedad, era muy difícil recuperar la salud; $12.1 \%$ dijo que los pacientes con cáncer no eran socialmente activos después del diagnóstico; $28.9 \%$, que las habilidades laborales de estos pacientes se reducían aun después de un tratamiento eficaz. Por otro lado, $33.6 \%$ de los participantes afirmó que los pacientes con cáncer eran fácilmente reconocibles por su apariencia; $29 \%$ estuvo de acuer- do o muy de acuerdo que los pacientes tenían dificultades sexuales; $71.4 \%$ creía que estos pacientes tenían que ser protegidos por la sociedad, y 7\% afirmó que los pacientes con cáncer no podrían hacer contribuciones a la sociedad. Por último, se observó que $7 \%$ de los participantes se sentía incómodo al estar con pacientes con esa enfermedad; $3.9 \%$ evitaría la interacción con ellos; $8.9 \%$ indicó que evitaría casarse con alguien cuyos familiares hubieran padecido cáncer; y 2.8\% afirmó que evitaría trabajar con personas que padecieran cáncer.

\section{Análisis factorial exploratorio (AFE)}

Dado que se observó una suficiente adecuación muestral $(\mathrm{KMO}=.791)$ y que la prueba de esfericidad de Bartlett resultaba significativa ( $X^{2}(66)$ $=1472.480 p<.001)$, se consideró viable realizar el AFE. A partir de la inspección de la gráfica de sedimentación y los resultados del análisis paralelo $\left(X^{2}(33), p<.001\right)$, se determinó la conveniencia de retener tres factores (Figura 1), para lo cual se generaron 1,000 conjuntos de datos aleatorios y se utilizó un intervalo de confianza (IC) de 95\%.

Figura 1. Gráfico de sedimentación con las datos reales y simulados de acuerdo al análisis paralelo.

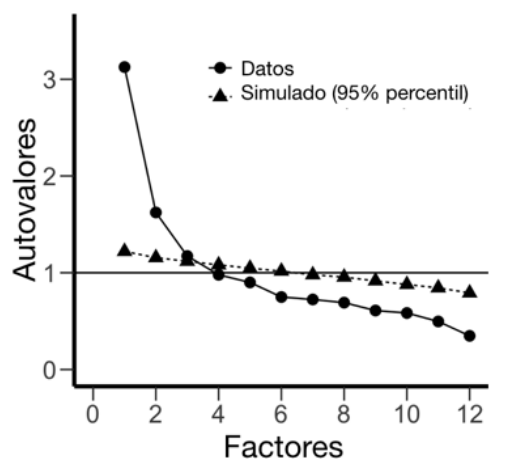

El primer factor (F1) obtuvo un valor Eigen de 3.126 y explicó $26.05 \%$ de la varianza; el segundo (F2), $13.523 \%$, con un valor Eigen de 1.623, y el último (F3), con un valor Eigen de 1.173, explicó 9.773\% del total de la varianza. Así, el instrumento alcanzó a explicar $49.346 \%$ de la misma. Las cargas factoriales de los ítems para los factores retenidos se determinaron en .40 (Tabla 2). 
Tabla 2. Cargas factoriales (Los valores en negritas indican la pertenencia a un mismo factor dentro de una misma columna. Puntuaciones sin negritas reflejan puntuaciones menores a .40 , por lo que no se agrupan en el factor).

\begin{tabular}{|c|c|c|c|}
\hline Ítem & Factor 1 & Factor 2 & Factor 3 \\
\hline 1 & .007 & -.138 & 0.541 \\
\hline 10 & .847 & -.087 & -.039 \\
\hline 11 & .591 & .051 & -.047 \\
\hline 12 & .776 & .012 & -.031 \\
\hline 2 & -.121 & -.026 & .631 \\
\hline 3 & .170 & .170 & .282 \\
\hline 4 & -.001 & .312 & .255 \\
\hline 5 & .057 & 496 & -.068 \\
\hline 6 & .125 & .644 & -.083 \\
\hline 7 & -.090 & .174 & -.017 \\
\hline 8 & .422 & .083 & .106 \\
\hline 9 & .682 & -.072 & -.068 \\
\hline
\end{tabular}

Esta solución mantiene la propuesta de tres factores reportada por Cho et al. (2013); sin embargo, uno de los ítems (8) se integró de diferente manera, y tres de ellos $(3,4$ y 7$)$ se eliminaron debido a que su carga factorial fue menor de .40 .
Las correlaciones entre factores fueron relativamente moderadas. La correlación más alta $(r=.416)$ fue entre F2 y F3, mientras que la menor $(\mathrm{r}=.366)$ fue para $\mathrm{F} 1 \mathrm{con} \mathrm{F} 3$, en tanto que F1 y F2 obtuvieron una $r=.403$.

\section{Análisis factorial confirmatorio (AFC)}

El AFC indicó un óptimo ajuste $\left(X^{2}=59.498(24)\right.$, $p<.001, X^{2} / \mathrm{DF}=2.479, \mathrm{RMSA}=.048, \mathrm{CFI}=.974$, $\mathrm{TLI}=.961, \mathrm{NFI}=.958)$.

\section{Análisis de confiabilidad}

El análisis de consistencia interna se llevó a cabo con los ítems resultantes del AFE. El coeficiente Omega para el total del instrumento fue de .738, y los coeficientes para cada factor, los siguientes: $\mathrm{F} 1=.891, \mathrm{~F} 2=.477$ y F3 $=491$.

\section{Datos normativos}

Se presentan en la Tabla 3 las medias, desviaciones típicas y percentiles que pueden usarse como indicadores normativos.

Tabla 3. Valores descriptivos.

\begin{tabular}{|c|c|c|c|c|c|c|c|c|c|c|c|c|}
\hline & \multicolumn{3}{|c|}{ F1 Discriminación } & \multicolumn{3}{|c|}{$\begin{array}{l}\text { F2 Estereotipos } \\
\text { pacientes } \\
\text { con cáncer }\end{array}$} & \multicolumn{3}{|c|}{$\begin{array}{l}\text { F3 Imposibilidad } \\
\text { de recuperación }\end{array}$} & \multicolumn{3}{|c|}{$\begin{array}{l}\text { Total de los tres } \\
\text { factores }\end{array}$} \\
\hline & M & $\mathbf{H}$ & $\mathbf{M} / \mathbf{H}$ & M & $\mathbf{H}$ & $\mathbf{M} / \mathbf{H}$ & $\mathbf{M}$ & $\mathbf{H}$ & $\mathbf{M} / \mathbf{H}$ & $\mathbf{M}$ & $\mathbf{H}$ & $\mathbf{M} / \mathbf{H}$ \\
\hline Media & 6.39 & 7.04 & 6.65 & 4.25 & 4.38 & 4.3 & 3.96 & 4.04 & 3.99 & 14.59 & 15.45 & 14.94 \\
\hline D.E. & 2.02 & 2.64 & 2.3 & 1.23 & 1.29 & 1.26 & 1.3 & 1.3 & 1.3 & 3.09 & 3.79 & 3.42 \\
\hline Percentil 25 & 5 & 5 & 5 & 3 & 4 & 4 & 3 & 3 & 3 & 13 & 13 & 13 \\
\hline Percentil 50 & 6 & 6 & 6 & 4 & 4 & 4 & 4 & 4 & 4 & 14 & 15 & 14 \\
\hline Percentil 75 & 7 & 8 & 7 & 5 & 5 & 5 & 5 & 5 & 5 & 16 & 17 & 17 \\
\hline
\end{tabular}

\section{DISCUSIÓN}

Los resultados del presente estudio permiten confirmar la viabilidad del modelo sugerido por Cho et al. (2013) respecto al estigma hacia los pacientes con cáncer, pero plantea interrogantes sobre su confiablidad. Contar con un instrumento en español que tenga alguna evidencia de validez resulta oportuno debido a la falta de instrumentos en este idioma sobre el referido problema, por lo que lo anterior es una contribución a su estudio.

El análisis factorial exploratorio y el confirmatorio indicaron agrupamientos de ítems en factores de forma similar a la escala original, salvo el ítem 8 ("Los pacientes con cáncer no podrían hacer contribuciones a la sociedad"). Sin embargo, el valor semántico de dicho ítem refleja, en opinión de los presentes autores, un sentido de dis- 
criminación y no de estereotipia, según había sido clasificado en la versión original del instrumento.

Los datos recolectados permiten caracterizar las actitudes de una muestra universitaria mexicana hacia los pacientes con cáncer e iniciar la construcción de baremos que pueden ayudar a determinar patrones de normalidad en poblaciones similares a la de la muestra estudiada. Debido a que las variables psicosociales interactúan con la cultura (Fu, Anderson, Courtney y Hu, 2007), disponer de normas que sirvan como marco de contrastación puede ser útil para entender mejor la forma en que un grupo poblacional enfrenta socialmente un padecimiento. Los datos hallados indican claramente que la muestra mexicana exhibe un patrón de respuesta diferente al reportado por Cho et al. (2013). Por ejemplo, la elección más alta de cada ítem en la muestra mexicana estudiada es marcadamente menor que en la muestra coreana (Cho et al., 2013). Aunque se han reportado similitudes en las actitudes de mexicoamericanos y coreano-americanos hacia la autonomía de pacientes oncológicos terminales (Blackhall y Murphy, 1995), los hallazgos matizan, e incluso quizá contradicen, tales similitudes. Una posible explicación podría radicar en una visión fatalista del cáncer reportado en las poblaciones asiáticas (Wong-Kim, Sun y DeMattos, 2003). Lo contrario se ha reportado en poblaciones mexicoamericanas (Skolarus et al., 2012). Si bien estos reportes son comparaciones con otras poblaciones con otras enfermedades, abre una vía de exploración posible.

Analizar las diferencias de género no fue propósito de este estudio; sin embargo, los datos indican una actitud más desfavorable en los hombres, al contrario de las mujeres, lo que es relativamente congruente con trabajos que han hallado que los hombres son menos propensos a mostrar actitudes positivas relacionadas con la enfermedad (Wendt y Shafer, 2016). Incluso cuando se trata de la pro- pia enfermedad, muestran actitudes menos favorables que las mujeres (Ritvo et al., 2013). Así, los presentes hallazgos confirman estas tendencias comportamentales.

A pesar de que la validez convergente del modelo de evaluación de estigma parece adecuada, es importante que en próximos estudios se revise la validez concurrente en cuanto que aquí se han aportado datos sobre el ajuste óptimo del modelo. Igualmente, es conveniente revisar la consistencia interna del instrumento. Si bien la confiabilidad de todo el instrumento es aceptable al tener un valor superior a 70 (Vera, Ávila y Dorantes, 2014), la confiablidad es baja en dos de los tres factores, los que únicamente están compuestos por dos ítems. Para factores con pocos ítems, son aceptables los valores moderados (Jackson y Verberg, 2006), sobre todo en las primeras versiones de un instrumento (Nunnally y Bernstein, 1995). Pese a ello, esos valores fueron inferiores a un valor moderado. Al considerarse valioso el modelo del instrumento para evaluar la estigmatización hacia los pacientes con cáncer, podría mejorarse en próximos estudios si se aumentara el número de ítems y se revisara además la estabilidad temporal.

Por último, es importante reiterar que la estigmatización hacia personas con cáncer afecta el bienestar de quienes sufren la enfermedad (Cho, Choi et al., 2013; Greidanus et al., 2018; Stergiou-Kita, Pritlove y Kirsh, 2016; Trusson y Pilnick, 2017), y por ello un primer paso para atender este problema es conocer la manera en que ocurre el fenómeno del estigma. La evaluación del instrumento aquí presentado permite establecer su viabilidad y sus posibilidades de mejora, y asimismo sirve de base para próximos estudios sobre este tópico, pues conocer las variables asociadas al bienestar psicológico de quien sufre el estigma permitirá realizar intervenciones más amplias, relevantes y oportunas.

\section{REFERENCIAS}

Berrenberg, J.L., Finlay, K.A., Stephan, W.G. y Stephan, C. (2002). Prejudice toward people with cancer or AIDS: Applying the integrated threat model. Journal of Applied Biobehavorial Research, 7(2), 75-86. doi: 10.1111/j.1751-9861.2002.tb00078.x.

Blackhall, L. y Murphy, S. (1995). Ethnicity and attitudes toward patient autonomy. JAMA: Journal of the American Medical Association, 274(10), 820.

Boulos, D. N. y Ghali, R. R. (2014). Awareness of breast cancer among female students at Ain Shams University, Egypt. Global Journal of Health Science, 6(1), 154. doi: 10.5539/gjhs.v6n1p154. 
Chapple, A., Ziebland, S. y McPherson, A. (2004). Stigma, shame, and blame experienced by patients with lung cancer: qualitative study. British Medical Journal, 328(7454), 1470. doi: 10.1136/bmj.38111.639734.7C.

Cho, J., Choi, E.K., Kim, S.Y., Shin, D.W., Cho, B.L., Kim, C.H. y Park, J.H. (2013). Association between cancer stigma and depression among cancer survivors: A nationwide survey in Korea. Psycho-Oncology, 22(10), 2372-2378. doi: 10.1002/ pon.3302.

Cho, J., Smith, K., Choi, E.K., Kim, I.R., Chang y. J., Park, H.Y., Guallar, E. y Shim Y.M. (2013). Public attitudes toward cancer and cancer patients: a national survey in Korea. Psycho-Oncology, 22, 605-613. doi: 10.1002/pon.3041.

Dany, M., Chidiac, A. y Nassar, A.H. (2015). Human papillomavirus vaccination: assessing knowledge, attitudes, and intentions of college female students in Lebanon, a developing country. Vaccine, 33(8), 1001-1007. doi: 10.1016/j.vaccine.2015.01.009.

Feng, L.-S., Li, X.-Y., Wang, H.-R., Zhan, J.-J., Chen, D. y Wang Y.-F. (2018). Development and validation of the Cancer Self-perceived Discrimination Scale for Chinese cancer patients. Health and Quality of Life Outcomes, 16(1), 165. doi: 10.1186/s12955-018-0984-x.

Fu, S., Anderson, D., Courtney, M. y Hu, W. (2007). The relationship between culture, attitude, social networks and quality of life in midlife Australian and Taiwanese citizens. Maturitas, 58(3), 285-295. doi: 10.1016/j.maturitas.2007.08.017.

Fujisawa, D. y Hagiwara, N. (2015). Cancer stigma and its health consequences. Current Breast Cancer Reports, 7(3), 143-150.

Greidanus, M.A., de Boer, A.M., de Rijk, A.E., Tiedtke, C.M., Dierckx de C., B., Frings-Dresen, M.W. y Tamminga, S.J. (2018). Perceived employer-related barriers and facilitators for work participation of cancer survivors: A systematic review of employers' and survivors' perspectives. Psycho-Oncology, 27(3), 725-733. doi: 10.1002/pon.4514.

Grigsby, T.J., Kent, E.E., Montoya, M.J., Sender, L.S., Morris, R.A., Ziogas, A. y Anton-Culver, H. (2014). Attitudes toward cancer clinical trial participation in young adults with a history of cancer and a healthy college student sample: a preliminary investigation. Journal of Adolescent and Young Adult Oncology, 3(1), 20-27. doi: 10.1089/jayao.2013.0030.

Hofmann-Broussard, C., Armstrong, G., Boschen, M.J. y Somasundaram, K.V. (2017). A mental health training program for community health workers in India: Impact on recognition of mental disorders, stigmatizing attitudes and confidence. International Journal of Culture and Mental Health, 10(1), 62-74. doi: 10.1080/17542863.2016.1259340.

Jackson, W. y Verberg, N. (2006). Methods: Doing social research. Toronto: Pearson Education.

Latif, R. (2014). Knowledge and attitude of Saudi female students towards breast cancer: A cross-sectional study. Journal of Taibah University Medical Sciences, 9, 328-334.

Lee, J.L. y Kim, K.S. (2011). The relationships between stigma, distress, and quality of life in patients with lung cancer. Journal of Korean Oncology Nursing, 11(3), 237-246.

López D., O., Canga A., N., Mujika, A., Pardavila B., M. I., Belintxon, M. y Serrano M., I. (2017). Five paradoxes in health promotion. Gaceta Sanitaria, 31(3), 269-272. doi: 10.1016/j.gaceta.2016.10.011.

Machado, G., Biancovilli, P. y Jurberg, C. (2017). Voices about a stigma: cancer in the opinion of three different segments in Brazilian society. Journal of Cancer Education, 32(2), 413-416. doi: 10.1007/s13187-015-0962-5.

Marlow, L.A. y Wardle, J. (2014). Development of a scale to assess cancer stigma in the non-patient population. BMC Cancer, 14(1), 285. doi: 10.1186/1471-2407-14-285.

Martínez M., G. (2008). Análisis de un instrumento de evaluación del afrontamiento del estrés en sujetos con cáncer. Revista Iberoamericana de Diagnóstico y Evaluación Psicológica, 25(1), 35-49.

McDonald, R.P. (2011). Test theory: A unified treatment. New York: Routledge.

Mosher, C.E. y Danoff-Burg, S. (2007). Death anxiety and cancer-related stigma: A terror management analysis. Death Studies, 31(10), 885-907. doi: 10.1080/07481180701603360.

Napier, A., Ancarno, C., Butler, B., Calabrese, J., Chater, A., Chatterjee, H. y Macdonald, A. (2014). Culture and health. The Lancet, 384(9954), 1607-1639. doi: 10.1016/S0140-6736(14)61603-2.

Nunnally, J.C. y Bernstein, I.H. (1995). Teoría psicométrica. México: McGraw-Hill.

Parker, R. (2012). Stigma, prejudice and discrimination in global public health. Cadernos de Saude Publica, 28(1), 164-169.

Peiró, G., Corbellas, C., Camps, C., Sánchez, P.T., Godes, M., D’Ambra, M. y Blasco, A. (2006). Estudio descriptivo sobre el proceso de comunicar el diagnóstico y el pronóstico en oncología. Psicooncología, 3(1), 9-17.

Reichenheim, M.E. y Moraes, C.L. (2007). Operacionalização de adaptação transcultural de instrumentos de aferição usados em epidemiologia. Revista de Saúde Publica, 41(4), 665-673. doi: 10.1590/S0034-89102006005000035.

Revelle, W. y Zinbarg, R.E. (2009). Coefficients alpha, beta, omega, and the glb: Comments on Sijtsma. Psychometrika, 74(1), 145-154.

Reyes L., I. y García B., L.F. (2008). Procedimiento de validación psicométrica culturalmente relevante: Un ejemplo. La Psicología Social en México, XII, 625-636.

Ritvo, P., Myers, R.E., Paszat, L., Serenity, M., Perez, D.F. y Rabeneck, L. (2013). Gender differences in attitudes impeding colorectal cancer screening. BMC Public Health, 13(1), 500. doi: 10.1186/1471-2458-13-500.

Rodríguez A., M.C. y Palacios E., X. (2013). Representaciones sociales del cáncer y de la quimioterapia. Psicooncología, 10(1), 79-93. doi: 10.5209/rev_PSIC.2013.v10.41949. 
Sambanje, M.N. y Mafuvadze, B. (2012). Breast cancer knowledge and awareness among university students in Angola. Pan African Medical Journal, 11(70)1-10.

Skolarus, L.E., Lisabeth, L.D., Sánchez, B.N., Smith, M.A., Garcia, N.M., Risser, J.M. y Morgenstern, L.B. (2012). The prevalence of spirituality, optimism, depression, and fatalism in a bi-ethnic stroke population. Journal of Religion and Health, 51(4), 1293-1305. doi: 10.1007/s10943-010-9438-4.

Stergiou-Kita, M., Pritlove, C. y Kirsh, B. (2016). The "Big C"-stigma, cancer, and workplace discrimination. Journal of Cancer Survivivorship, 10(6), 1035-1050.

Trusson, D. y Pilnick, A. (2017). Between stigma and pink positivity: women's perceptions of social interactions during and after breast cancer treatment. Sociology of Health \& Illness, 39(3), 458-473. doi: 10.1111/1467-9566.12486.

Vera J., J.A., Ávila G., M.E. y Dorantes A., G. (2014). Manual de evaluación de la victimización y percepción de la violencia, delincuencia e inseguridad, y la confianza en las instituciones. Cuernavaca (México): UAEM.

Vrinten, C., Gallagher, A., Waller, J. y Marlow, L.A.V. (2019). Cancer stigma and cancer screening attendance: a population based survey in England. BMC Cancer, 19(1), 566. doi: 10.1186/s12885-019-5787-x.

Wendt, D. y Shafer, K. (2016). Gender and Attitudes about mental health help seeking: Results from national data. Health and Social Work, 41(1), 20-28. doi: 10.1093/hsw/hlv089.

Wong-Kim, E., Sun, A. y DeMattos, M.C. (2003). Assessing cancer beliefs in a Chinese immigrant community. Cancer Control, $10(5), 22-28$.

World Medical Association (2013). Declaration of Helsinki. Ethical principles for medical research involving human subjects. Ferney-Voltaire (Francia): WMA. Recuperado de: https://www.wma.net/policies-post/wma-declaration-of- helsinki- ethical- principles- for- medical- research- involving- human- subjects/. 